\section{Epidemiologia dos acidentes provocados por linhas com cerol: estudo de vítimas atendidas em hospital de trauma em Belo Horizonte, Brasil}

\author{
Epidemiology of powdered glass- \\ coated kite line injuries: Victims \\ admitted to a trauma hospital in \\ Belo Horizonte, Brazil
}

Roberto Marini Ladeira'

Paulo Roberto Lima Carreiro ${ }^{1,111}$

João Baptista de Rezende-Neto"

Gabriel Correia lannuzzi"'

André Alves Elias"'I

' Hospital João XXIII da FHEMIG, Belo Horizonte, Minas Gerais.

"Faculdade de Medicina da Universidade Federal de Minas Gerais.

III Faculdade de Medicina da UNIFENAS, Belo Horizonte, Minas Gerais.

\section{Resumo}

Objetivo: Descrever as características das vítimas de acidentes por linhas com cerol atendidas em hospital de trauma de Belo Horizonte, Brasil. Métodos: Estudo seccional com vítimas de acidentes por linhas com cerol no período de 2005 a 2009. Os dados foram coletados retrospectivamente nos prontuários médicos. Foram utilizadas medidas de tendência central e comparação entre proporções para descrever as características das vítimas. Resultados: Foram identificadas 211 vítimas no período estudado, $94 \%$ das quais eram do sexo masculino. Houve concentração do atendimento nos meses de junho e julho (37\% e $41,7 \%$, respectivamente). A média de idade foi de 15 anos, não havendo diferença entre os sexos. No conjunto das vítimas, os membros superiores foram o local mais prevalente de lesões, mas entre os motociclistas e ciclistas os principais locais de lesão foram o pescoço e a face. Foram registrados 2 óbitos $(0,95 \%)$, ambos em motociclistas e causados por lesões das estruturas anatômicas do pescoço. O local de residência das vítimas se concentrou em distritos sanitários de Belo Horizonte (Leste, Nordeste e Centro-Sul). Conclusão: Apesar da benignidade das lesões identificadas no período estudado, podem ocorrer lesões fatais, principalmente em motociclistas. Medidas educativas e preventivas são necessárias para evitar a ocorrência de lesões potencialmente fatais.

Palavras-chave: Epidemiologia. Linha com cerol. Ferimentos e lesões. Morbidade. Mortalidade. Serviço hospitalar de emergência. 


\section{Abstract}

Objective: To describe the characteristics of victims of powdered glass-coated (manja) kite line injuries admitted to a trauma hospital in Belo Horizonte, Brazil. Methods: Cross-sectional study of victims with powdered glass-coated kite line injuries between 2005 and 2009. Data were obtained retrospectively from medical records. Central tendency and comparison of ratios were used to characterize victims. Results: 211 victims were admitted between 2005 and 2009, $94 \%$ of which males. The majority of cases occurred in June and July (37\% e $41.7 \%$, respectively). Mean age was 15 years, with no difference between men and women. For the group of victims as a whole, upper limbs were the most prevalent site of injury, although neck and face injuries were the main site of injury among motorcyclists and cyclists. Two deaths $(0.95 \%)$ were identified, both in motorcyclists, due to injury to neck structures. Residences were concentrated in three administrative areas (East, Northeast and South-central). Conclusion: Although the majority of injuries recorded were not severe, fatal injuries can occur, mainly among motorcyclists. Educational and preventive measures are needed to avoid potentially fatal injuries.

Keywords: Epidemiology. Kite-line. Morbidity. Mortality. Wounds and injuries. Hospital emergency service.

\section{Introdução}

O hábito de soltar pipas é uma atividade de lazer muito popular e difundida em diversos países do mundo, incluindo o Brasil, praticada principalmente por crianças e jovens. Entretanto, esta atividade tem sido relacionada à ocorrência de lesões graves e até óbitos em virtude de eletrocussão na rede elétrica, quedas e lesões causadas pela linha ${ }^{1-4}$, especialmente quando utilizado o cerol, uma mistura artesanal de cola e vidro moído que é adicionada às linhas das pipas. Esta prática tem como objetivo tornar a linha da pipa mais resistente e capaz de cortar as linhas de outras pipas, numa brincadeira conhecida como batalha de pipas.

Em países asiáticos, os festivais de pipas são atividades culturais e de lazer muito difundidas e uma linha semelhante ao cerol é conhecida com o nome de Manja. Somente no ano de 2005, no Paquistão, cerca de 500 pessoas se feriram e 19 morreram em decorrência de lesões relacionadas à prática de soltar pipas ${ }^{3}$. Na maioria dos casos, o agente causador foi o Manja, sendo que quedas, choques elétricos, agressões e acidentes de trânsito também foram causas de lesões e mortes.

Existem poucos trabalhos na literatura em saúde a respeito das lesões provocadas diretamente pela linha com cerol, sendo que alguns se referem a outros mecanismos de lesões relacionados à atividade de soltar pipas, especialmente às provocadas por descargas elétricas ${ }^{1-4}$. Neste trabalho estudamos a ocorrência das lesões provocadas especificamente pelo uso do cerol nas linhas, avaliando características das vítimas, gravidade e localização das lesões, ocorrência de internação hospitalar e óbitos.

Esperamos que o conhecimento destes dados possa orientar medidas preventivas eficazes resultando na redução das lesões e óbitos relacionadas ao uso do cerol em linhas utilizadas para empinar pipas.

\section{Metodologia}

Foram identificadas todas as vítimas de 
lesões causadas por linhas de cerol admitidas na Unidade de Emergência do Hospital João XXIII-FHEMIG (Fundação Hospitalar do Estado de Minas Gerais), referência para atendimentos de urgência e emergência na Região Metropolitana de Belo Horizonte, com cerca de 4,8 milhões de habitantes.

Foram incluídos pacientes cujo atendimento ocorreu entre janeiro de 2005 e dezembro de 2009. Mediante revisão de prontuários foram extraídos dados referentes à vítima (sexo, idade, endereço, tipo), ao dia da semana, mês e ano da ocorrência, à descrição do acidente e das lesões apresentadas; à localização anatômica das lesões, à necessidade de internação hospitalar; ao tipo de alta.

Os dados foram analisados de forma descritiva, utilizando-se distribuição de frequência e teste t para comparação de médias e qui-quadrado para comparação de proporções. Foi utilizado para construção do banco de dados e análise o software EPI INFO for Windows 3.5.1.

O estudo foi aprovado pelo Comitê de Ética em Pesquisa da FHEMIG, sob o protocolo $068 / 2009$. Os autores declaram não haver conflito de interesses no presente trabalho.

\section{Resultados}

De janeiro de 2005 a dezembro de 2009 foram atendidos 509.547 pacientes vítimas de trauma, dentre os quais foram identificadas $211(0,04 \%)$ vítimas de lesões por linhas com cerol. No ano de 2005 deu-se a maior proporção $(28 \%)$ desses acidentes.

Com relação ao mês de ocorrência, julho $(41,7 \%)$, junho $(37,0 \%)$ e janeiro $(10,4 \%)$, concentraram a maior parte dos atendimentos, com alternância dos casos entre esses dois primeiros meses, ao longo dos anos (Figura 1).

A quase totalidade das vítimas (94\%) era do sexo masculino. A idade variou entre $3 \mathrm{e}$ 67 anos, com média de 19,1 anos e mediana de 15 anos, sem diferença estatisticamente significativa entre os sexos (Teste $t=0,92$; $\mathrm{p}=0,358)$.

Cento e cinquenta e duas vítimas $(86,1 \%)$ eram moradoras de Belo Horizonte, $19(12,9 \%)$ residiam em municípios vizinhos e apenas dois (1\%) eram de regiões mais distantes.

O local de ocorrência dos acidentes não estava disponível. No que se refere ao endereço das vítimas em Belo Horizonte, 26,7\% eram de bairros do distrito sanitário Leste, 25,0\% do distrito sanitário Nordeste e 20,0\% do distrito sanitário Centro - Sul (Figura 2).

As lesões ocorreram mais frequentemente nos membros superiores $(57,3 \%)$, principalmente nos dedos. Em seguida, o pescoço $(15,6 \%)$, a cabeça $(12,8 \%)$ e os membros inferiores $(11,4 \%)$ foram os

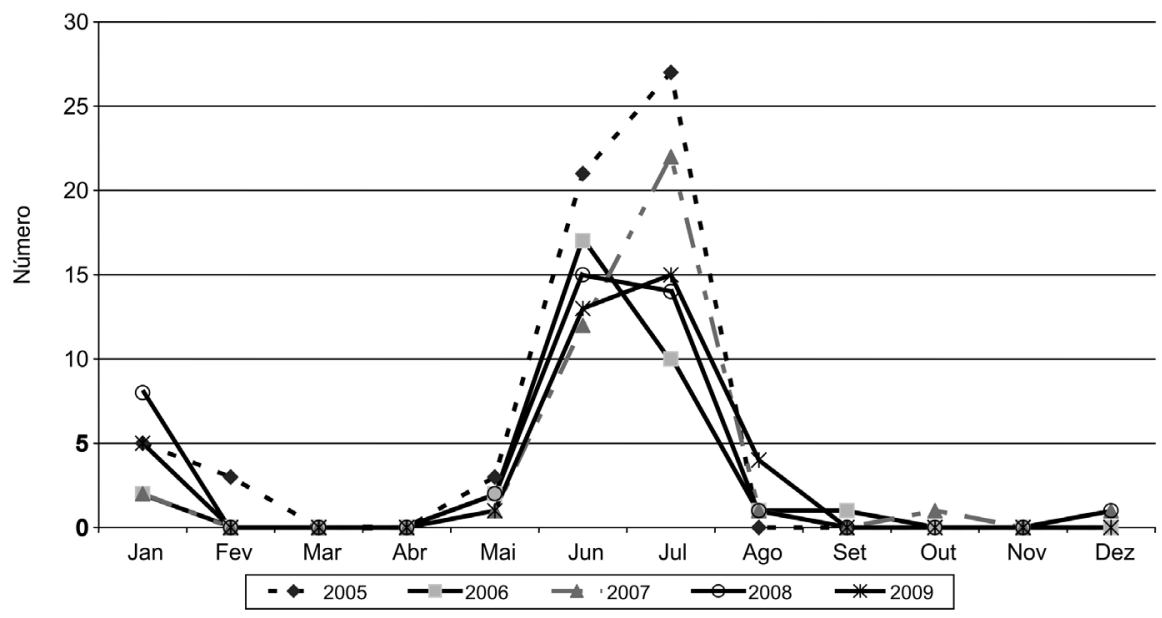

Figura 1 - Atendimentos de vítimas de lesões provocadas por linha com cerol, 2005 a 2009.

Figure 1 - Emergency room visits of victims injured by glass-coated kite lines, 2005-2009. 


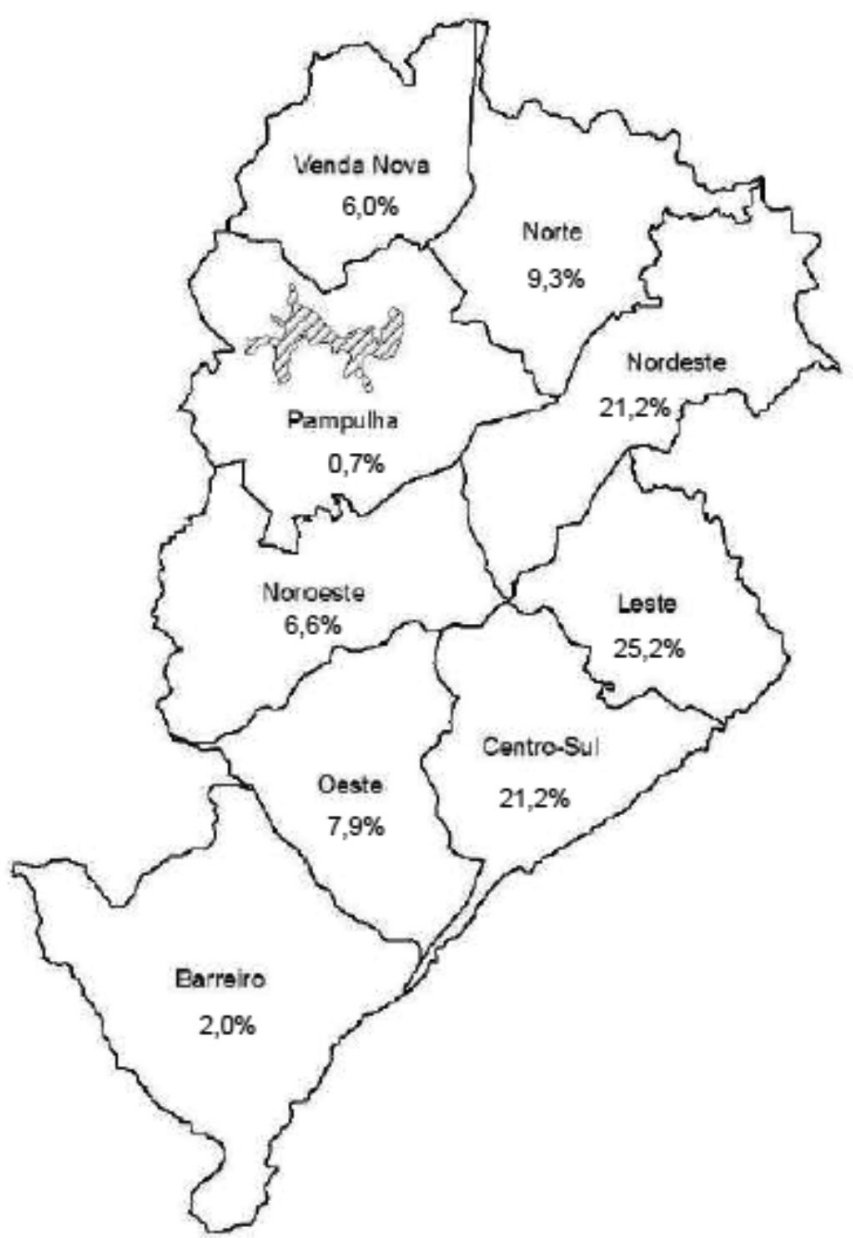

Figura 2 - Vítimas de lesões por linha com cerol, por distrito de residência, Belo Horizonte, 2005 a 2009.

Figure 2 - Victims of kite line injuries, by district of residence, Belo Horizonte, 2005-2009.

segmentos mais acometidos (Figura 3).

Devido à insuficiência de dados presentes no prontuário, só foi possível identificar o tipo de vítima em 33,6\% dos casos. Destes, $55 \%$ foram identificados como motociclistas, $8,5 \%$ como ciclistas, $16,9 \%$ como a pessoa que empinava pipa e $9,8 \%$ como pedestres.

Considerando-se apenas os indivíduos identificados como motociclistas, a topografia das lesões foi: pescoço $(48,7 \%)$, face/cabeça (33,3\%) e membros superiores $(12,8 \%)$. Entre os ciclistas, $50 \%$ das lesões foram no pescoço e $50 \%$ na face/cabeça. As vítimas que empinavam pipa apresentaram, em sua totalidade, lesões cortantes dos dedos da mão. Com relação às vítimas identificadas como pedestres, o predomínio foi de lesões nos dedos das mãos e das pernas.

As lesões em membros superiores foram mais frequentes nos indivíduos com menos de 20 anos. Entre 20 e 39 anos a localização mais frequente foi o pescoço. A face também foi um local frequente de lesões nesta faixa etária (Figura 4). A análise da localização das lesões nas vítimas maiores de 60 anos ficou prejudicada, dado o pequeno número (3).

De todas as vítimas, $14,0 \%$ foram internadas, com média de permanência de 2 dias e mediana de 1 dia. Entre as vítimas que foram internadas, as principais lesões foram as que acometeram as estruturas mais profundas do pescoço $(24,0 \%)$ e também as lesões de membros que acometeram tendões $(48,3 \%)$ (Tabela 1). Foram registrados 2 óbitos (0,95\%), ambos em motociclistas que 


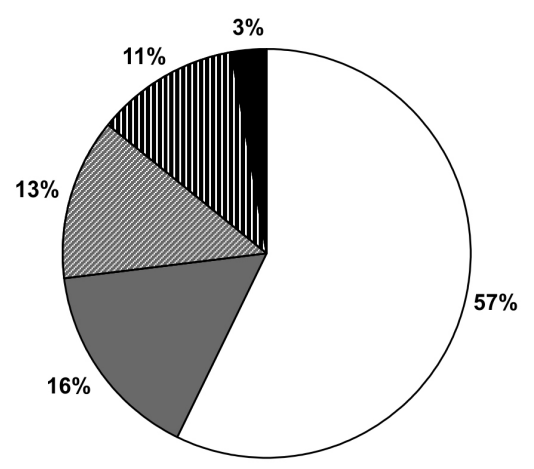

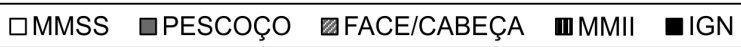

Figura 3 - Distribuição das lesões por linha com cerol, por topografia, 2005-2009.

Figure 3 - Anatomical distribution of kite line injuries, 2005-2009.

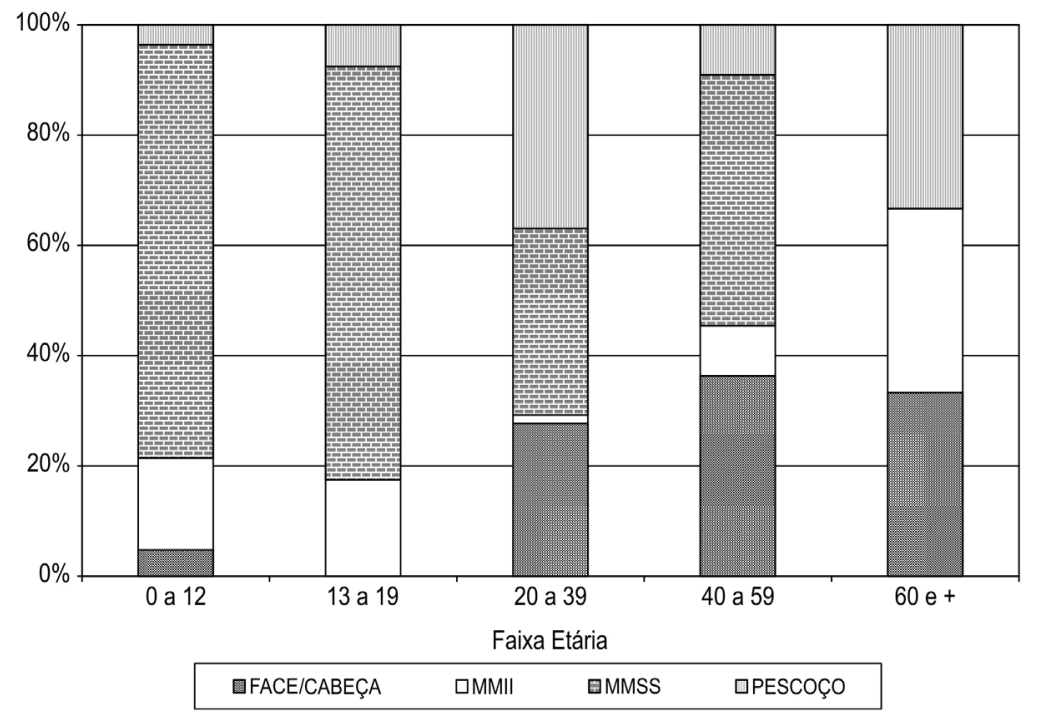

Figura 4 - Topografia das lesões por linha de cerol, por faixa etária, 2005-2009.

Figure 4 - Anatomical distribution of kite line injuries by age, 2005-2009.

sofreram lesões na região cervical, sendo um com lesão bilateral de carótida e de traqueia e o outro com lesão bilateral de veia jugular.

\section{Discussão}

Os meses de maior ocorrência das lesões por cerol foram junho e julho (80\%), coincidindo com o inverno, período com mais ventos e, portanto, mais propício à prática de soltar pipas ${ }^{4}$. Apesar de julho também ser um mês de férias escolares, permitindo que as crianças tenham mais tempo livre para as brincadeiras, as férias não parecem ser determinantes, pois o número de atendimentos nos meses de dezembro é pequeno.

Quanto ao local de residência das vítimas, observamos uma concentração em Belo Horizonte e, principalmente, em bairros de quatro regiões ao leste da cidade. Esta distribuição geográfica pode estar relacionada a uma maior frequência de ventos na época que concentra a maior parcela dos acidentes ${ }^{5}$ e ao fato de serem regiões cuja ocupação ainda é predominantemente horizontal (casas ou edifícios com número menor de andares). Além disso, em alguns bairros dessas regiões predomina uma 
Tabela 1 - Lesões por linha com cerol, por região anatômica e regime de tratamento, Belo Horizonte, 2005 a 2009

Table 1 - Anatomical distribution of kite line injuries, by type of treatment, Belo Horizonte, 2005-2009

\begin{tabular}{|c|c|c|c|c|}
\hline & & Internação & Ambulatorial & Total \\
\hline \multirow[t]{7}{*}{ Face/Cabeça } & Nariz & 0 & 10 & 10 \\
\hline & Orelha & 0 & 5 & 5 \\
\hline & Glabela & 0 & 4 & 4 \\
\hline & Palpebra/olho & 0 & 5 & 5 \\
\hline & Lábios & 0 & 1 & 1 \\
\hline & Não especificada & 0 & 1 & 1 \\
\hline & Sub-total & 0 & 26 & 26 \\
\hline \multirow[t]{5}{*}{ Pescoço } & Lesão superficial & 2 & 23 & 25 \\
\hline & Lesão camada muscular & 3 & 2 & 5 \\
\hline & Lesão cartilagem cricóide & 0 & 1 & 1 \\
\hline & Lesão veia jugular e/ou traquéia & 4 & 0 & 4 \\
\hline & Sub-total & 9 & 26 & 35 \\
\hline \multirow[t]{8}{*}{ Membros superiores } & Dedo(s) & 2 & 94 & 96 \\
\hline & Dedo(s) - lesão de tendão & 11 & 4 & 15 \\
\hline & Dedo(s) - lesão de tendão, vaso e nervo & 2 & 0 & 2 \\
\hline & Mão & 0 & 4 & 4 \\
\hline & Ante-braço & 0 & 3 & 3 \\
\hline & Ante-braço - lesão de tendão & 1 & 0 & 1 \\
\hline & Ombro & 0 & 1 & 1 \\
\hline & Sub-total & 16 & 106 & 122 \\
\hline \multirow[t]{8}{*}{ Membros inferiores } & Perna & 1 & 11 & 12 \\
\hline & Perna - lesão de tendão & 1 & 1 & 2 \\
\hline & Perna - lesão muscular & 1 & 0 & 1 \\
\hline & Pé & 0 & 2 & 2 \\
\hline & Pé - lesão de tendão & 1 & 1 & 2 \\
\hline & Dedo(s) & 0 & 2 & 2 \\
\hline & Coxa & 0 & 1 & 1 \\
\hline & Sub-total & 4 & 18 & 22 \\
\hline Não informada & & 0 & 6 & 6 \\
\hline Total & & 29 & 182 & 211 \\
\hline
\end{tabular}

população de menor renda, nas quais as crianças ainda preservam o hábito de empinar pipas. Apesar de não estar disponível a informação sobre o local de ocorrência do acidente, consideramos razoável supor, exceto no caso dos ciclistas e motociclistas, que os acidentes tenham ocorrido em local perto da residência das vítimas.

O predomínio de lesões acometendo indivíduos do sexo masculino (94\%) se justifica pelo hábito de soltar pipas ser mais difundido entre os meninos.

A maioria das lesões acomete a própria pessoa que manuseia o cerol durante a confecção da linha, ou ao soltar a pipa, tal como demonstrado por Rezende-Neto e cols ${ }^{4}$. Nestes casos, as lesões mais frequentes ocorrem nos dedos das mãos e outras regiões dos membros superiores. Tal fato parece justificar a maior concentração de lesões nos membros superiores em indivíduos até 19 anos. Pessoas que estão próximas a quem está empinando pipas e pedestres também podem ser atingidas, porém as lesões mais graves são observadas em ciclistas e, principalmente, em motociclistas, que são atingidos pela linha de cerol enquanto estão em movimento.

A velocidade de deslocamento e a velocidade do vento são fatores determinantes para a gravidade das lesões. Nos motociclistas, as lesões mais graves ocorrem na região 
cervical, por ser uma área desprotegida e exposta ao contato com a linha. Neste estudo, os óbitos ocorreram em dois motociclistas admitidos com ferimento cervical profundo associado a lesões da traqueia, de veias jugulares e de artérias carótidas, bilateralmente. Devido à gravidade dessas lesões, alguns pacientes morrem no local do acidente e não são contabilizados nas estatísticas hospitalares. Por este motivo, acredita-se que os óbitos por lesões por cerol em motociclistas e ciclistas acontecem com maior frequência do que a observada em casuísticas que analisam apenas os pacientes atendidos em hospitais.

O estudo apresenta algumas limitações devidas ao fato de utilizar informações constantes em prontuários médicos, que habitualmente apresentam muitas falhas de registro. Deste modo, a identificação do tipo de vítima, da atividade que a vítima fazia no momento em que ocorreu a lesão e do local de ocorrência do acidente ficou prejudicada. Pode haver concentração de atendimentos de pacientes cuja região de moradia fique mais próxima ao hospital. Além disso, pode haver sub-registro de acidentes com lesões mais leves, que não procuram atendimento.

Com o objetivo de se tentar prevenir estas lesões principalmente em motociclistas, foi colocado no mercado um dispositivo de proteção composto por hastes metálicas com um gancho em sua extremidade, instaladas no guidão das motocicletas, o que, no caso de contato com a linha de cerol, impede que o condutor seja atingido (Figura 5). Está em tramitação atualmente no Congresso Nacional projeto de lei que obriga as fábricas e montadoras de motocicletas a instalar a antena de proteção contra fios cortantes ${ }^{6}$.

No Brasil, vários Estados e municípios possuem leis que proíbem a utilização do cerol $^{7-9}$, mas as ações de fiscalização são praticamente inexistentes, o que pode explicar o número semelhante de casos em cada ano do período estudado.

Outro fator que propicia a difusão desta prática é que o cerol é um produto

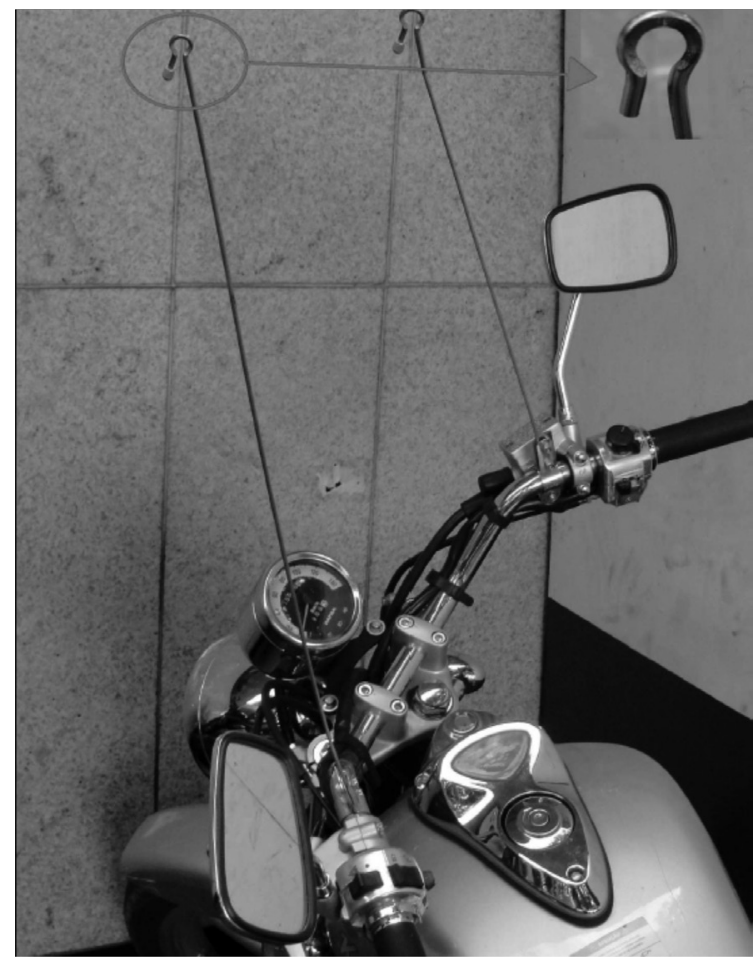

Figura 5 - Antena instalada em motocicleta (no detalhe, o gancho).

Figure 5 - Antenna installed on a motorcycle (see hook in detail). 
de fabricação caseira, de baixo custo, e os materiais utilizados na sua fabricação são de fácil acesso. Curiosamente, já existe um mercado paralelo até na internet, onde sítios de anúncios classificados ou sítios de discussão sobre pipas oferecem linhas "industrializadas" mais sofisticadas e com um poder de corte muito maior que o das linhas de fabricação caseira ${ }^{10}$. Uma das mais conhecidas é a "linha chilena", fabricada com o acréscimo de produtos químicos (óxido de alumínio e óxido de silício).

Neste cenário, a adoção de medidas socioeducativas mais eficazes e de medidas regulatórias contra a venda de linhas industrializadas são fundamentais para a prevenção da ocorrência de lesões provocadas por linhas com cerol.

Como os dados do presente estudo mostraram uma grande concentração das lesões em moradores de regiões específicas da cidade de Belo Horizonte, este achado pode ser um elemento facilitador para o desenvolvimento de ações educativas, nas escolas e outros espaços sociais e comunitários, visando crianças e pais, com objetivo de estimular a prática segura de soltar pipas, sem cerol e também evitando o contato com linhas elétricas e quedas de lajes e telhados.

Também é necessário reforçar a importância da utilização das antenas de proteção por motociclistas como forma de reduzir o risco de lesões na região cervical, potencialmente fatais.

Mais estudos e com maior abrangência são necessários para identificar a real importância das lesões por linhas com cerol no Brasil.

\section{Referências}

1. Tiwari VK, Sharma D. Kite-flying: a unique but dangerous mode of electrical injury in children. Burns 1999; 25: 537-9.

2. Wandhede AG, Sariya DR. An electrocution by metal kite line. Forensic Science International 2006; 163: 141-3.

3. Wandhede AG, Sariya DR. "Manja" - a dangerous threat. J Forensic Leg Medicine 2008; 15: 189-92.

4. Rezende Neto JB; Ferreira GC; Silva Filho AL; Fontes MOBQ; Bomfim F; Abrantes WL. Kiting Injuries: Report of Two Cases and Discussion. J Trauma 2000; 48: 310-1.

5. Prudente CN, Raia A, Reis RJ. Comportamento do Vento na Região Metropolitana de Belo Horizonte. In Anais do XIV Congresso Nacional de Meteorologia: Florianópolis; 2006.

6. Câmara Federal do Brasil - Projeto de Lei No 6.378 de 2005. Dispõe sobre a obrigatoriedade de inclusão pelas fábricas e montadoras de motocicletas, de antena de proteção contra fios cortantes como equipamento de segurança.
7. Estado de São Paulo, Lei No 12.192, de 06 de janeiro de 2006. Dispõe sobre a proibição do uso de cerol ou de qualquer produto semelhante que possa ser aplicado em linhas de papagaios ou pipas. Diário Oficial do Estado de São Paulo, 07 de janeiro de 2006.

8. Estado de Minas Gerais - Lei No 14349 de 15 de julho de 2002. Dispõe sobre a proibição do uso de pipas com linha cortante em áreas públicas comuns (Regulamentada pelo Decreto 43585, de 15/09/2003. Minas Gerais Diário do Executivo, 16 de setembro 2003.

9. Município de Belo Horizonte - LEI No 8. 563, de 13 de maio de 2003, Dispõe sobre a proibição de utilizar cerol em linhas para empinar pipa. Diário Oficial do Município de Belo Horizonte, 14 de maio de 2003.

10 http://g1.globo.com/Noticias/SaoPaulo/0,,MUL6810585605,00-LINHAS+IMPORTADAS+ COM+CEROL+E+F ERIAS+AUMENTAM+ACIDENTES+COM+PIPAS.html [Acessado em 19 de fevereiro de 2011]

Recebido em: 01/05/2011

Versão final apresentada em: 07/10/2011

Aprovado em: 28/11/2011 